Check for updates

Cite this: RSC Adv., 2017, 7, 20152

\title{
Green preparation of a cellulose nanocrystals/ polyvinyl alcohol composite superhydrophobic coating
}

\begin{abstract}
Jingda Huang, ${ }^{\text {ab }}$ Shaoyi Lyu, ${ }^{a}$ Feng Fu, ${ }^{a}$ Yan Wu ${ }^{b c}$ and Siqun Wang (DD *ab
Organic solvents, which are often used in the preparation process of superhydrophobic coatings, are volatile and toxic. The inorganic particles also widely used in their preparation are difficult to degrade, which may result in environmental contamination. In this study, commercial lignin-coated cellulose nanocrystal ( $\mathrm{L}-\mathrm{CNC}$ ) particles, which are environmentally friendly and biodegradable and formed by CNC solution containing a small amount of lignin in a spray-drying process, uniting strength with a rough surface, were used to provide a good foundation for a superhydrophobic coating due to the different size and irregular surfaces of the particles. A superhydrophobic coating is successfully prepared by spraying a L-CNC/polyvinyl alcohol (PVA) composite paint and then modifying it via chemical vapor deposition (CVD). The resulting coating has not only good superhydrophobicity and self-cleaning properties, but also excellent abrasion resistance. In addition, no organic solvents or inorganic particles were used in the preparation process. Thus, this coating could potentially be used in some applications that require nontoxicity or environmental safety such as food packaging.
\end{abstract}

Received 2nd December 2016 Accepted 3rd April 2017

DOI: $10.1039 / c 6 r a 27663 f$

rsc.li/rsc-advances the environment. Especially in the most spray methods, the paint is first prepared using organic solvents and then sprayed onto the substrate surface. ${ }^{15-17}$ The organic solvents evaporate into air and pollute the environment, especially when spraying is done indoors, where relatively confined spaces have poor ventilation. In preparation process of superhydrophobic coatings, ethanol, ${ }^{18}$ tetrahydrofuran, ${ }^{19}$ acetone, ${ }^{20}$ toluene, ${ }^{21}$ and so on were common organic solvents which were toxic or flammable and explosive substances. In addition, nonbiodegradable inorganic particles such as $\mathrm{SiO}_{2},{ }^{22} \mathrm{TiO}_{2},{ }^{23} \mathrm{CaCO}_{3},{ }^{24} \mathrm{Fe}_{3} \mathrm{O}_{4}{ }^{25}$ and so on are often used to build rough surface structure.

Previously, our research group successfully used nanofibrillated cellulose (NFC) to prepare a superhydrophobic coating by CVD after spraying, ${ }^{26}$ but anhydrous ethanol was used in preparation process. In this study, we successfully avoided using any organic solvents and inorganic particles in preparing a superhydrophobic coating. Lignin-coated cellulose nanocrystals (L-CNC) ${ }^{27}$ particles, which were produced by CNC solution containing a little lignin with less hydrophilic than CNC in spray-drying and possessed right micro-nano structure, were used as the raw material to build rough surface structure required as a necessary for a superhydrophobic coating. L-CNC particles possess many good properties, being environmentally friendly and mainly plant-derived, and have much higher strength than NFC due to its higher crystallinity. ${ }^{28,29}$ Some studies have reported using $\mathrm{CNC}$ as a reinforcing material, ${ }^{29-31}$ and researchers have sought ways to make CNC disperse in a polymer matrix in order to give full play to its ability to improve strength. ${ }^{28}$ Even though CNC could be dispersive in 


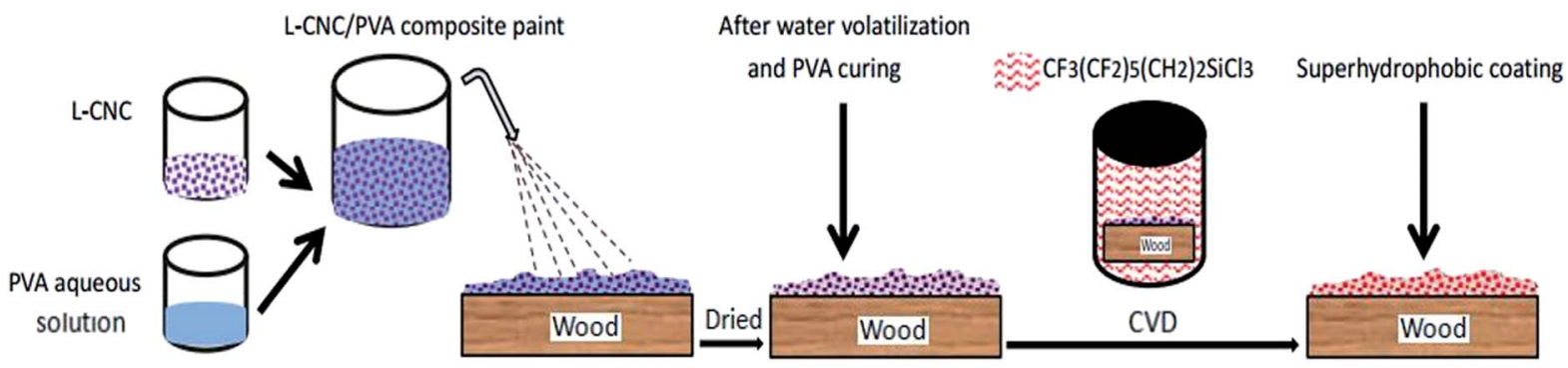

Fig. 1 Sketch of the procedure to prepare L-CNC/PVA composite superhydrophobic coating on wood substrate.

aqueous solution due to its surface charge, ${ }^{32}$ it is difficult to prepare a dried dispersive CNC due to its tendency toward easy agglomeration. But in this research, a dispersive CNC was not desired. Instead, L-CNC particles were very satisfactorily used to build rough surface structure for a superhydrophobic coating. To increase adhesion between the substrate and the coating, polyvinyl alcohol (PVA), a nontoxic water-soluble polymer, was used as a binder. Ultimately, this green preparation process produced a superhydrophobic coating that could be applied in fields such as food packaging, where nontoxicity is desirable.

As shown in Fig. 1, the preparation process was uncomplicated. First, L-CNC particles were added to a PVA water solution to form a composite paint. Then the paint was sprayed onto the wood surface and dried at room temperature or in an oven. Finally, low surface free energy modification was conducted via CVD. It is worth noting that the concentration of PVA was key. Too low a concentration would lead to poor adhesion, but too high a concentration would cause PVA to become viscous and inconvenient for spraying. As prepared, not only was the coating environmentally friendly, but it also showed good self-cleaning properties and abrasion resistance.

\section{Experiment}

\section{Materials}

Polyvinyl alcohol (PVA) (white particles, 99+\% hydrolyzed, typical average $M_{\mathrm{w}} 85000-124000$ ) was purchased from Sigma Aldrich, USA. Lignin-coated cellulose nanocrystals (L-CNC, $4.3 \%$ moisture, a brown powder made from CNC solution, lignin content 3-6 wt\%), were purchased from American Process Inc., USA. $1 H, 1 H, 2 H, 2 H$-Perfluorooctyltrichlorosilane $\quad\left(\mathrm{CF}_{3}\left(\mathrm{CF}_{2}\right)_{5}(-\right.$ $\left.\mathrm{CH}_{2}\right)_{2} \mathrm{SiCl}_{3}, 97 \%$ ) as a low energy reagent was purchased from Sigma-Aldrich, USA. Chemical reagents were not further processed and were directly used as received from the manufacturers. Small pieces of wood of dimensions $4.5 \mathrm{~cm} \times 2.5 \mathrm{~cm} \times$ $1.5 \mathrm{~cm}$ were chosen as the substrates.

\section{Preparation of L-CNC/PVA composite superhydrophobic coating}

$20 \mathrm{~g}$ of PVA particles were weighted and added to $180 \mathrm{~g}$ of distilled water at $80^{\circ} \mathrm{C}$, then stirred with a magnetic stirrer for $500 \mathrm{rad} \mathrm{min}^{-1}$ for 5 hours until completely melted. The pre-PVA solution was divided into five different concentrations of 0.5 ,
1.0, 1.5, 2.0 and $2.5 \mathrm{wt} \%$, respectively, with distilled water. $3 \mathrm{~g} \mathrm{~L}$ CNC particles were then added to $47 \mathrm{~g}$ of PVA solution at the different concentrations and then stirred for 1 hour to form a homogeneous paint mixture. The paint mixture $(0.25-0.35 \mathrm{~g}$ spray quantity) was then sprayed using an airbrush (Uxcell Mini 0.5 K3 HVLP Gravity Feed Paint Spray Gun Airbrush) onto the wood surface and then placed at room temperature for over 5 hours or dried for above $10 \mathrm{~min}$ at $103{ }^{\circ} \mathrm{C}$ in an oven until moisture evaporation was complete. The above woods were then put into a $500 \mathrm{ml}$ bottle, which included, inside, a smaller $30 \mathrm{ml}$ bottle with $0.8 \mathrm{~g}$ of low-energy modifier. The $500 \mathrm{ml}$ bottle was sealed with a cap and placed in a $90{ }^{\circ} \mathrm{C}$ oven for above 5 hours. Finally, the samples were removed from the oven and left for $30 \mathrm{~min}$ at $90{ }^{\circ} \mathrm{C}$ to remove unreacted low-energy material and by-products of the CVD process.

\section{Characterization}

A Zeiss Auriga SEM/FIB crossbeam workstation (Germany) using an accelerating voltage of $3 \mathrm{kV}$ was used to observe the surface morphology of the different samples. Fourier transform infrared spectroscopy (FTIR, Perkin Elmer, USA) was chosen to detect the chemical composition of the L-CNC/PVA composite coatings before and after modification by CVD at a scanning range of 4000-600 $\mathrm{cm}^{-1}$, scanning 16 times.

The water contact angle (WCA) was measured using a drop shape analysis system (EasyDrop, Germany, Kruss) at ambient temperature. The slide angle (SA) was measured using a selfmade SA measuring station (as shown in Fig. 2a), as reported in our previous paper. ${ }^{26}$ In brief, a protractor with a hole in its baseline center was vertically fixed by adhesive to a strip of aluminum with a hole in its section center and the two holes were aligned. A " $\mathrm{T}$ " iron frame fixed with a smooth panel was inserted into the overlapping holes and could be rotated $180^{\circ}$. A level measurement instrument was used to adjust the smooth panel before each measurement. A water droplet of $4 \mu \mathrm{L}$ was used for each measurement of WCA and SA, and each WCA or SA was recorded as the average value of five measurements at random points.

The self-cleaning properties of the coatings were tested in the following manner. Some dust water was poured onto the sample tilted at an angle of $5^{\circ}$, and the surface condition of the sample was then observed. In another test, some dust was first put onto the surface of the sample, which was, at the same, 


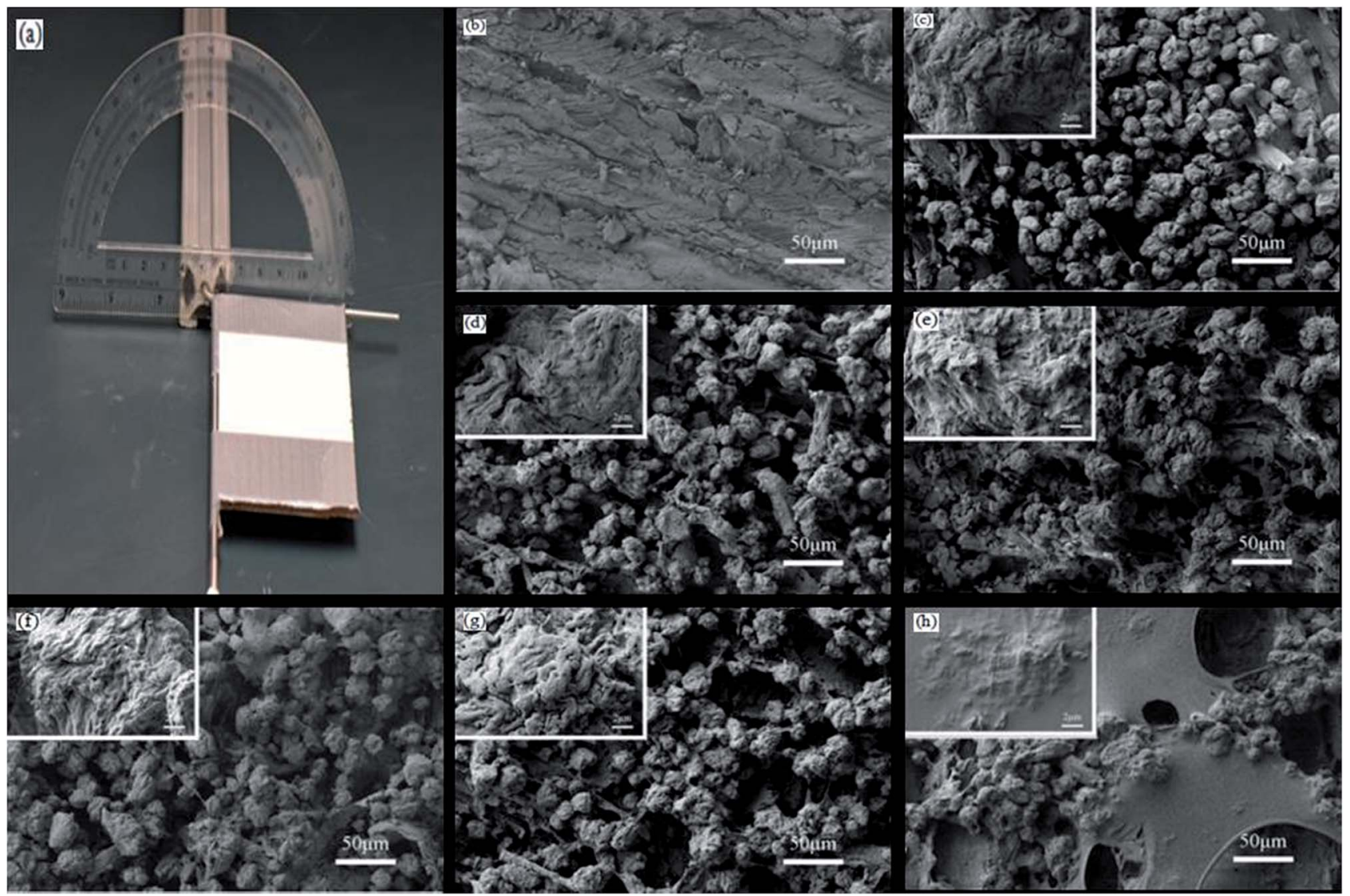

Fig. 2 (a) Self-made slide angle measuring station; surface morphology of (b) pristine wood surface, (c) pure L-CNC coating and highmagnification picture of its particle surface; composite coating with (d) $0.5 \mathrm{wt} \%$, (e) $1 \mathrm{wt} \%$, (f) $1.5 \mathrm{wt} \%$, (g) $2 \mathrm{wt} \%$ and (h) $2.5 \mathrm{wt} \%$ PVA concentration and high magnification images of particles surfaces, respectively.

tilted at an angle of $5^{\circ}$, and then water droplets were increasingly dripped on the upper portion of the sample surface to observe the surface condition.

The surfaces were also tested for abrasion resistance. As the method that has been previously reported, ${ }^{5}$ the treated side of the sample was placed against a piece of sandpaper (1500 grit) and weighted using a $100 \mathrm{~g}$ of weight. The sample was pushed by external force along a $10 \mathrm{~cm}$ straight path, followed by being horizontally shifted $90^{\circ}$ and pushed for another $10 \mathrm{~cm}$ length. This was denoted as one sandpaper abrasion cycle.

\section{Results and discussions}

\section{Surface morphology of coatings}

As shown in Fig. 2b, after being sanded, the pristine wood surface was not smooth and some uneven grooves appeared, which were from broken wood cells. As shown in Fig. 2c, the pure L-CNC particles were of different sizes and irregular shapes and formed from the irregular aggregation of the CNC solution droplets in a spray-drying process. Sprayed onto the wood surface, most of the pure L-CNC particles overlapped with each other and formed micro-rugged structures. As shown in the highly magnified image in Fig. $2 \mathrm{c}$, the pure L-CNC particles surfaces existed different uneven gaps and protuberances that provided nanostructural roughness. Thus the L-CNC particles and their surfaces formed the micro-nano hierarchical structure that was necessary for the superhydrophobic surface. But some of the L-CNC particles also fell into the grooves and could not play a role in building the rough protuberances that provided roughness. The adhesion between the L-CNC particles and the wood would be also very poor. Thus it could be foreseeable that using PVA to cover the wood surface acted not only to fill the grooves but also would provide adhesion between the L-CNC particles and the wood. As shown in Fig. $2 \mathrm{~d}-\mathrm{g}$, all the coating morphologies had no much difference during the PVA concentration range of $0.5-2.0 \mathrm{wt} \%$. But compared with pure $\mathrm{L}-$ CNC particles, the L-CNC particles mixed with PVA were in closer contact with each other due to formation of PVA film among particles. Therefore, each particle mixed with PVA would be like a "sticky ball"; particles stuck together, with the effect of stabilizing the micro-nano hierarchical structure to some extent. The grooves were difficult to distinguish because wood surfaces were also covered by PVA, which would be helpful for adhesion between the particles and wood surface. At $2.5 \mathrm{wt} \%$ PVA concentration, a large area of PVA film was formed and covered a part of the coating. As shown in these high magnification images in Fig. $2 \mathrm{~d}-\mathrm{g}$, even though they were covered by PVA, the surfaces of the L-CNC particles still appeared to have different uneven gaps and protuberances and the nanostructure roughness wasn't highly altered in the PVA concentration range. 
But as shown in Fig. 2h, at 2.5 wt\% PVA concentration, some particles surfaces were almost completely covered in some areas of the coating and had become smooth.

\section{Wettability and self-cleaning properties}

As shown in Fig. 3a, the pristine wood surface without any treatment was hydrophilic. Fig. 3b-g show the wettability of the different samples treated with low energy reagent. Fig. 3 b shows though the pristine wood surface had a certain roughness, it was insufficient to provide enough roughness for the formation of a superhydrophobic coating, and the WCA was only $142.6^{\circ}$. The water droplets' adhesion was high and the SA was greater than $70^{\circ}$, and the water droplets were difficulty to roll away. As shown in Fig. 3c-e, the woods surfaces that had been covered with L-CNC/PVA composite paint in the PVA concentration range of $0.5-1.5 \mathrm{wt} \%$ showed excellent superhydrophobic properties. As shown in Fig. 3i, the WCAs were $157.3^{\circ}, 158.7^{\circ}$ and $158.2^{\circ}$, respectively and all SAs were less than $10^{\circ}$, allowing water droplets to form spheres and roll easily, having benefited from the micro-nano hierarchical structures formed by stacking of the particles and their rough surface morphology. Although the L-CNC particles were covered by PVA film in the composite coatings, the PVA film was not thick enough to completely cover the L-CNC particles in the PVA concentration range of $0.5-1.5 \mathrm{wt} \%$, so the superhydrophobic properties showed no obvious change. The PVA could support adhesion, but too high a PVA concentration would affect the WCA. As shown in Fig. 3f, at $2.0 \mathrm{wt} \%$ PVA concentration, the WCA of the coating only reached $152.5^{\circ}$; the SA was $12^{\circ}$ because the PVA film was thick enough to affect the coating's roughness. As shown in Fig. $3 \mathrm{~g}$, when the PVA concentration was continuously increased to $2.5 \mathrm{wt} \%$, the WCA decreased to only $139^{\circ}$ and was not able to reach superhydrophobicity because many L-CNC particles were covered by PVA and lost too much roughness.

To investigate transparency of the composite coating, the wood surface was first marked with the letters "CRC.UT" before spraying. When the spray quantity was confirmed, the
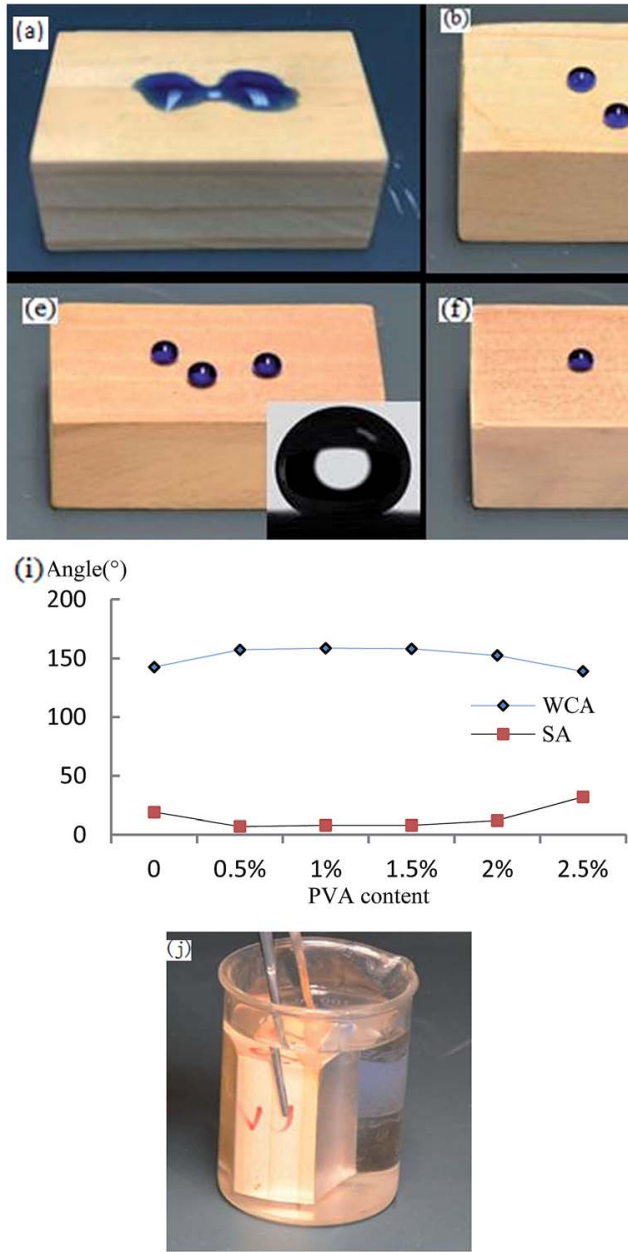
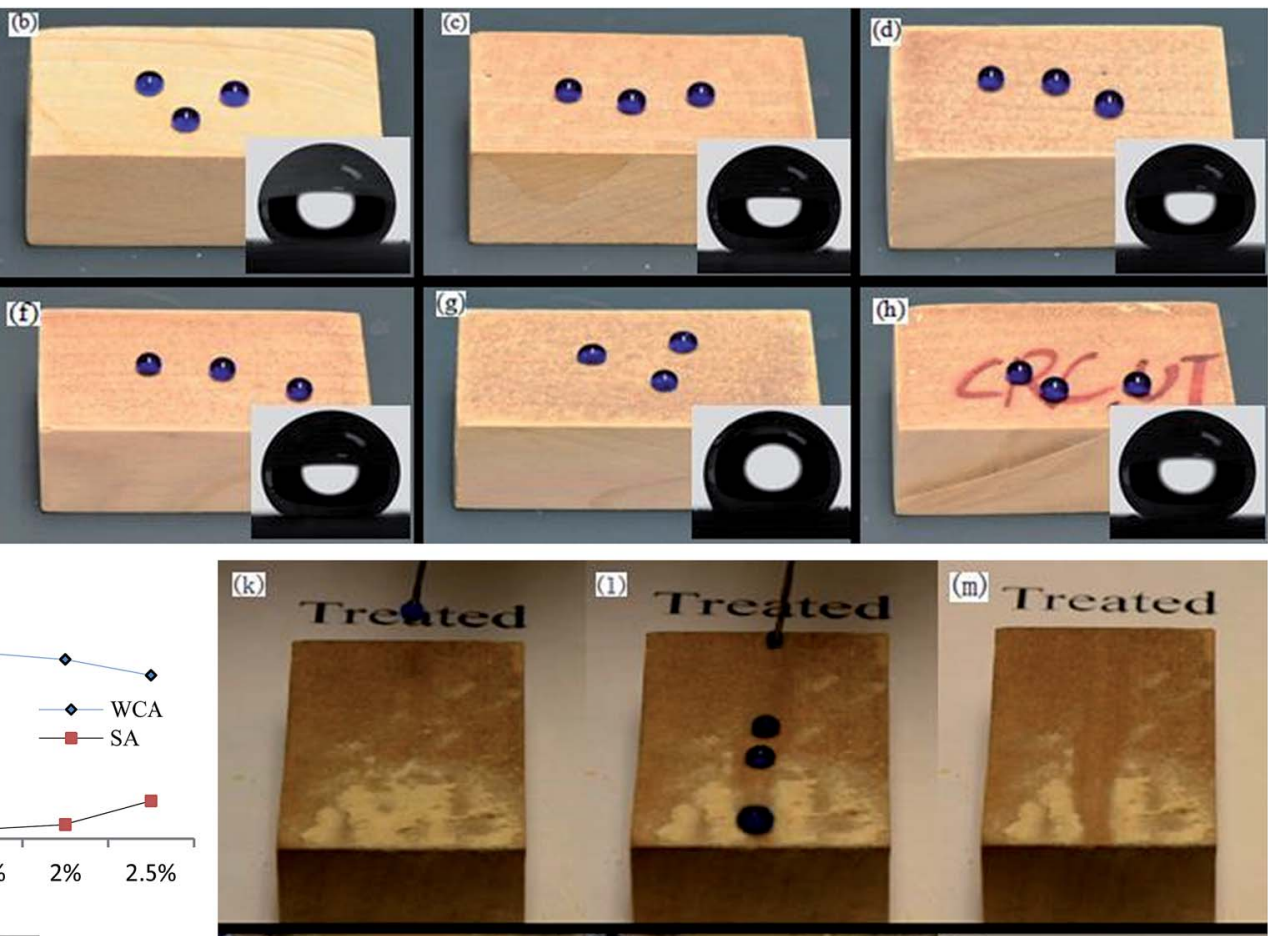

(m) Treated
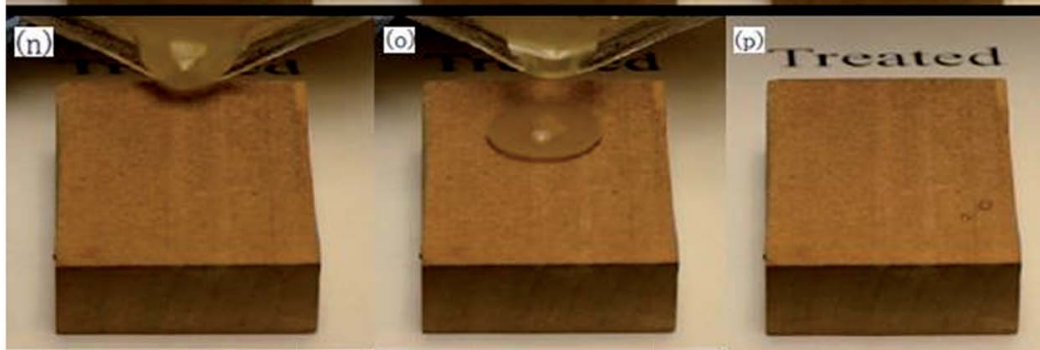

Fig. 3 Water droplets (dyed blue with methylene) status on the surfaces of (a) pristine wood without treatment, (b) modified pristine wood, LCNC/PVA composite coating with (c) $0.5 \mathrm{wt} \%$, (d) $1.0 \mathrm{wt} \%$, (e) $1.5 \mathrm{wt} \%$, (f) $2.0 \mathrm{wt} \%$ and (g) $2.5 \mathrm{wt} \%$ PVA concentration; (h) transparency of the treated L-CNC/PVA composite superhydrophobic coating; (i) change curve of WCA and SA of the coatings with different PVA content, (j) silver mirror phenomenon of the treated L-CNC/PVA composite superhydrophobic coating; self-cleaning test of L-CNC/PVA composite superhydrophobic coating using $(k-m)$ dust and $(n-p)$ dust water. 
transparency of the coating was associated with the L-CNC particles concentration. With the $0.25-0.35 \mathrm{~g}$ spray quantity, as shown in Fig. 3h, all of the coating showed as yellow brown, and the letters could be seen easily. This was because the PVA film was transparent and the L-CNC particles were not dense under uninfluenced superhydrophobic performance and hadn't completely covered the wood surface. The good translucency was attributed to the reflection of light in those areas that hadn't been covered by L-CNC particles.

The coating sample was clamped with a tweezer and fully immersed in water. When the glancing angle was larger than the critical angle, ${ }^{33}$ shown in Fig. 3j, the coating showed silver mirror phenomenon. But the untreated surface did not. It was known from the Cassie wetting statement that in the interface between a superhydrophobic surface and water, there was a layer of air that reflected light and created the silver mirror phenomenon, as we saw on this coating.

Self-cleaning is an important characteristic of a superhydrophobic coating and benefits from there being only a small contact area between water droplets and superhydrophobic surface due to the existence of the micro-nano hierarchical structure and the low surface free energy. Thus, in this test, water droplets easily rolled on the superhydrophobic surface. As shown in Fig. 3k-m, some dust was put onto the superhydrophobic surface. When water droplets were dripped onto the surface, the dust was attached to surfaces of the water droplets and taken away with the droplets. The roll trace was clean. Or when dusty water was poured onto the superhydrophobic surface tilted at the same angle (as shown in Fig. $3 n-p)$, the mixture of dust and water was able to form a sphere and roll away without contaminating the surface. While if on the untreated wood surface, the dusty water would stay on the surface and contaminated more due to water spread.

The coating was able to achieve superhydrophobicity in addition to good roughness, addition to good roughness, but low surface free energy modification was necessary. Fig. 4 shows the FTIR curve of the L-CNC/PVA composite coating before and after modification. The new peak at $867 \mathrm{~cm}^{-1}$ was caused by stretching vibrations of the $\mathrm{Si}-\mathrm{O}$ bonds resulted from reaction between $1 H, 1 H, 2 H, 2 H$-perfluorooctyltrichlorosilane and hydroxyl on the coating surface. That affirmed low energy

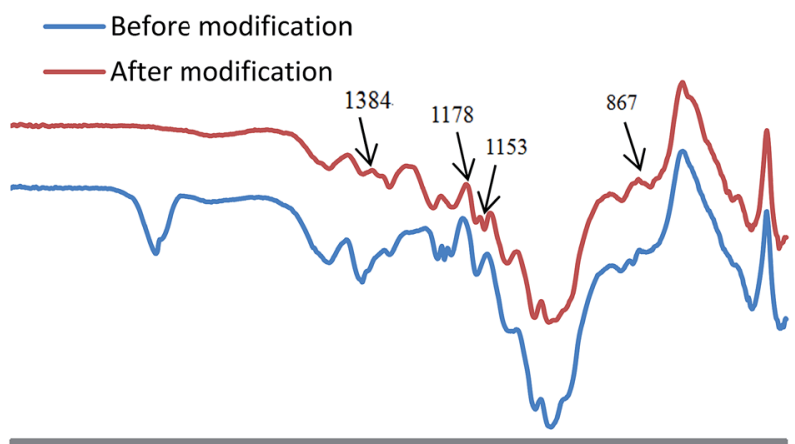

20001900180017001600150014001300120011001000900800700600

Fig. 4 FTIR spectra of the L-CNC/PVA composite coating before and after modification. material could be affixed to the coating surface by CVD. The absorption peak at 1153, 1178 and $1348 \mathrm{~cm}^{-1}$, were formed by the stretching vibrations of the $\mathrm{C}-\mathrm{F}$ bonds, which supplied low surface free energy.

\section{Sandpaper abrasion test}

Mechanical abrasion resistance is a key index for superhydrophobic surfaces. Especially in applications that need hard substrates, mechanical abrasion resistance directly affects the lifespan of superhydrophobic coatings. A common method for testing mechanical abrasion resistance is sandpaper abrasion, which not only can gauge the adhesion between a superhydrophobic coating and its substrate, but also can provide information about the coating's resistance to damage to the micro-nano rough structure of the coating's surface. If there is poor adhesion between a superhydrophobic coating and its substrate, sandpaper abrasion is likely to make the coating fall directly off the substrate. Conversely, if there is good adhesion, sandpaper abrasion can detect how the rough structure strengthens the coating surface. The demonstration of sandpaper abrasion for one cycle is shown in Fig. 5a and b.

\section{Influence of different PVA concentrations on abrasion resistance}

When the L-CNC particles concentration was $6 \mathrm{wt} \%$, as shown in Fig. 5c, the pure L-CNC particles coating was easily wiped away after only one cycle and lost superhydrophobicity. But the WCA was still $145^{\circ}$. That was because although L-CNC particles had been erased, the wood surface itself had a certain roughness. Those areas that hadn't been covered by L-CNC particles were also modified in the CVD process, causing them to demonstrate a certain hydrophobic performance. With continuing sandpaper abrasion, the wood surface would become hydrophilic when the treated wood areas were erased. Fig. 5i shows the influence of the coatings with different PVA concentrations on the abrasion resistance of superhydrophobic coatings under the same L-CNC particles concentration. Compared with the pure L-CNC particles coating, the abrasion resistance of all the L-CNC/PVA composite coatings were better. After one abrasion cycle, the composite coatings still possessed excellent superhydrophobic properties such that water droplets could still form spheres and roll easily away. That proved the PVA could supply good adhesion between the L-CNC particles and the wood and could fix the L-CNC particles to the wood surface to a certain extent (as shown in Fig. 5d). During the 0.5$1.5 \%$ PVA concentration, the abrasion cycles of the coatings increased with the increase of the PVA concentration. That was because in the concentration range, the higher the PVA concentration was, the more thicker the PVA film formed at the same spray quantity was, which led to the greater cladding of the L-CNC particles. In other words, the greater the adhesion was, the more abrasion cycles could be conducted. Fig. 5e-h show the surface morphology of the coatings with $0.5,1.0,1.5$ and $2.0 \mathrm{wt} \%$ PVA concentrations, respectively, on which the abrasion test was conducted until the WCA was less than $150^{\circ}$. As the PVA concentration increased, the amount of the 

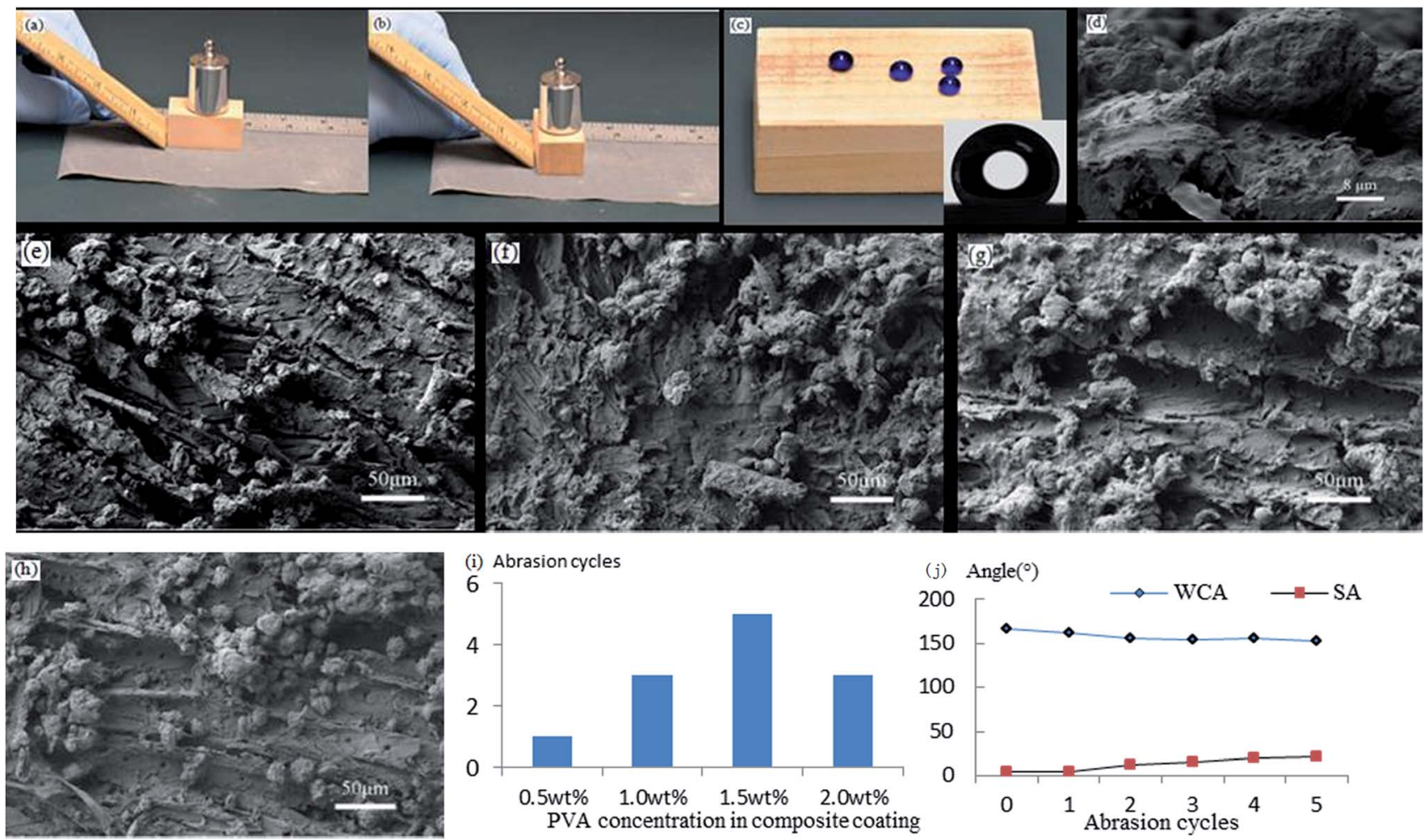

Fig. 5 (a and b) Schematic demonstration of one abrasion cycle; (c) surface status of pure L-CNC particles coating after one abrasion cycle; (d) high magnification image of the contact area between particles and wood surface; surface morphology of the coatings with (e) 0.5 wt\%, (f) 1.0 wt\%, (g) 1.5 wt\% and (h) 2.0 wt\% PVA concentration after abrasion to WCA below 150\%; (i) bar charts of abrasion cycles of the coatings with different PVA concentrations; (j) change curve of WCA and SA of the coating with 1.5 wt\% PVA concentration in abrasion process.

remaining L-CNC particles in a unit area increased, and the wood surface became fuzzier due to the thicker PVA film covering. But it could be seen that the greater PVA concentration couldn't support more abrasion cycles. At $2.0 \mathrm{wt} \%$ PVA concentration, the abrasion cycles diminished. Because before sandpaper abrasion, the L-CNC particles had been covered by the thick PVA film and their surface roughness was decreased, creating relatively poorer superhydrophobic properties than these coating with the lower PVA concentration. Even though there was higher adhesion between the particles and the wood surface, the coating was unable to withstand more abrasion cycles. Fig. 5 j shows the change curve of the WCA and the SA of the coating with $1.5 \mathrm{wt} \%$ PVA concentration during the abrasion process. The WCA became gradually lower and the SA increased due to the reduction in the roughness of the coating.

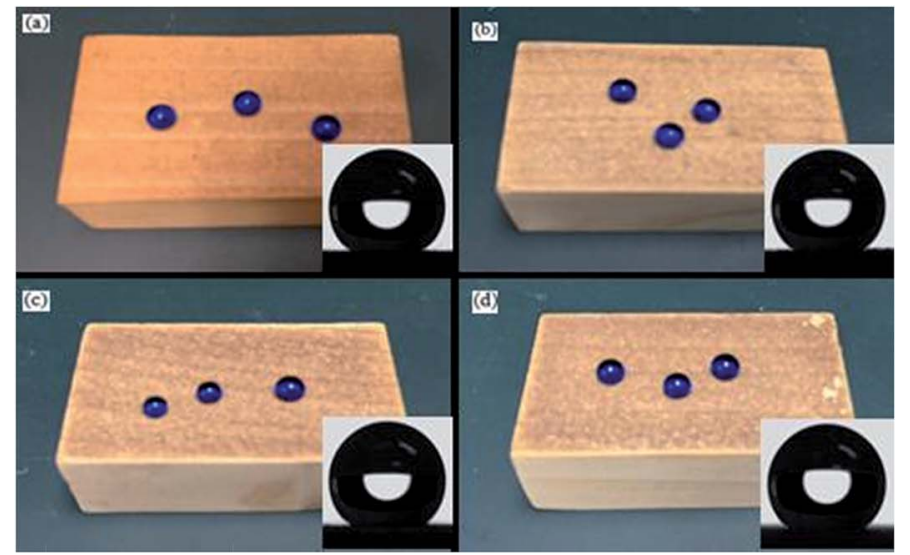

(e) Abrasion cycles

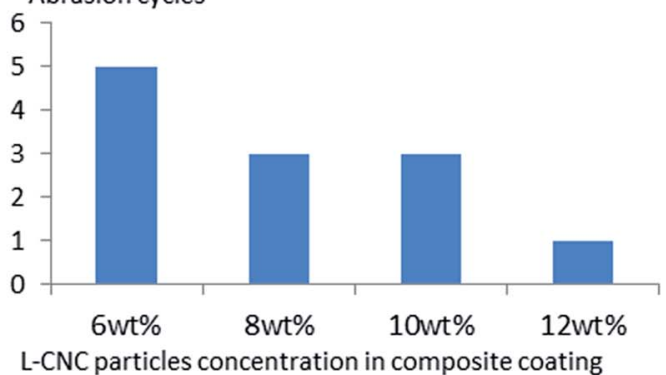

Fig. 6 Surface status of the coatings with L-CNC particles concentrations of (a) 6 wt\%, (b) 8 wt\%, (c) 10 wt\%, and (d) 12 wt\%; (e) bar charts of abrasion cycles of the coatings with different L-CNC particles concentration. 


\section{Influence of different particles concentrations on abrasion resistance}

As is known, under the same spray quantity, too low a concentration of particles impedes the building of a coating's roughness. When PVA concentration was firmed at $1.5 \mathrm{wt} \%$, the samples with four different particles concentration $(6,8,10$ and $12 \mathrm{wt} \%)$ were prepared. As shown in Fig. $6 \mathrm{a}-\mathrm{d}$, the coating color became darker as the particles concentration increased and all the WCAs were above $150^{\circ}$. The particles concentration not only had an effect on the superhydrophobicity and transparency of the coating, but also affected its abrasion resistance. Fig. 6e shows the relationship between different particles concentrations and abrasion cycles. The abrasion cycles went down as the particles concentration increased for two reasons. First, under the same spray quantity, the larger the particles concentration, the thicker the coating, and the greater its internal stress; this led to the coating adhesion being smaller and more easily worn off. Secondly, given a certain amount of PVA, the larger the particles concentration, the thinner the PVA film covering each particle surface and the lower the adhesion offered by the PVA film, so the ability to resist abrasion was smaller.

\section{Conclusions}

A L-CNC/PVA composite superhydrophobic coating can be easily prepared by using L-CNC particles to build a rough structure and PVA as the adhesive between the L-CNC particles and the substrates, then being modified with $1 \mathrm{H}, 1 \mathrm{H}, 2 \mathrm{H}, 2 \mathrm{H}$-perfluorooctyltrichlorosilane. The preparation process included forming the L-CNC/PVA composite paint through a uniform mixture of L-CNC particles and PVA aqueous solution and then spraying it onto the substrates surface, finally reducing the surface free energy by CVD. The resulting superhydrophobic coating not only showed outstanding self-cleaning properties, but also possessed good abrasion resistance that could withstand 5 sandpaper abrasion cycles. In addition, any organic solvent or inorganic particle hadn't been involved in the preparation process. This coating has great potential to be used in some areas that have high safety requirements such as food packaging.

\section{Acknowledgements}

This work was financially supported by the Special Fund for Forest Scientific Research in the Public Welfare (No. 201504603), Tennessee Experimental Station Project \#TEN00422 and the Special Fund of Chinese Central Government for Basic Scientific Research Operations in Commonweal Research Institutes (No. CAFINT2014K02). We thank the Department of Food Science \& Technology, the University of Tennessee for providing the use of the Drop Shape Analysis System.

\section{References}

1 H. Li, Y. Zhao and X. Yuan, Soft Matter, 2013, 9, 1005-1009. 2 M. Zhang and C. Wang, Carbohydr. Polym., 2013, 96, 396402.
3 M. W. England, C. Urata, G. J. Dunderdale and A. Hozumi, ACS Appl. Mater. Interfaces, 2016, 8, 4318-4322.

4 Y. Tang, Q. Zhang, X. Zhan and F. Chen, Soft Matter, 2015, 11, 4540-4550.

5 Y. Lu, S. Sathasivam, J. Song, C. R. Crick, C. J. Carmalt and I. P. Parkin, Science, 2015, 347, 1132-1135.

6 N. Wang, D. Xiong, Y. Deng, Y. Shi and K. Wang, ACS Appl. Mater. Interfaces, 2015, 7, 6260-6272.

7 G. Gong, K. Gao, J. Wu, N. Sun, C. Zhou, Y. Zhao and L. Jiang, J. Mater. Chem. A, 2015, 3, 713-718.

8 H. Zhou, H. Wang, H. Niu, A. Gestos, X. Wang and T. Lin, Adv. Mater., 2012, 24, 2409-2412.

9 X. Zhou, Z. Zhang, X. Xu, F. Guo, X. Zhu, X. Men and B. Ge, ACS Appl. Mater. Interfaces, 2013, 5, 7208-7214.

10 X. F. Zhang, R. J. Chen, Y. H. Liu and J. M. Hu, J. Mater. Chem. A, 2016, 4, 649-656.

11 Y. Lu, J. Song, X. Liu, W. Xu, Y. Xing and Z. Wei, ACS Sustainable Chem. Eng., 2012, 1, 102-109.

12 I. Sas, R. E. Gorga, J. A. Joines and K. A. Thoney, J. Polym. Sci., Part B: Polym. Phys., 2012, 50, 824-845.

13 Y. Zhao, Z. Xu, X. Wang and T. Lin, Appl. Surf. Sci., 2013, 286, 364-370.

14 S. S. Latthe and A. V. Rao, Surf. Coat. Technol., 2012, 207, 489-492.

15 H. Ogihara, J. Xie, J. Okagaki and T. Saji, Langmuir, 2012, 28, 4605-4608.

16 Y. Tang, J. Yang, L. Yin, B. Chen, H. Tang, C. Liu and C. Li, Colloids Surf., A, 2014, 459, 261-266.

17 J. Zhang, Z. Song, B. Li, Q. Wang and S. Wang, Desalination, 2013, 324, 1-9.

18 Z. Yuan, H. Chen, J. Tang and D. Zhao, J. Appl. Polym. Sci., 2009, 113, 1626-1632.

19 K. Tu, X. Wang, L. Kong, H. Chang and J. Liu, RSC Adv., 2016, 6, 701-707.

20 L. Zheng, Z. Li, S. Bourdo, K. R. Khedir, M. P. Asar, C. C. Ryerson and A. S. Biris, Langmuir, 2011, 27, 99369943.

21 H. Mertaniemi, A. Laukkanen, J. E. Teirfolk, O. Ikkalaa and R. H. A. Ras, RSC Adv., 2012, 2, 2882-2886.

22 D. A. Schaeffer, G. Polizos, D. B. Smith, D. F. Lee, S. R. Hunter and P. G. Datskos, Nanotechnology, 2015, 26, 055602 .

23 Y. Zhao, Y. Liu, Q. Xu, M. Barahman and A. M. Lyons, ACS Appl. Mater. Interfaces, 2015, 7, 2632-2640.

24 G. G. Wang, L. Q. Zhu, H. C. Liu and W. P. Li, Langmuir, 2011, 27, 12275-12279.

25 B. Wang, Y. Liu, Y. Zhang, Z. Guo, H. Zhang, J. H. Xin and L. Zhang, Adv. Mater. Interfaces, 2015, 2, 1500234.

26 J. Huang, S. Lyu, F. Fu, H. Chang and S. Wang, RSC Adv., 2016, 6, 106194-106200.

27 Y. Peng, D. J. Gardner and Y. Han, Cellulose, 2012, 19, 91102.

28 B. L. Peng, N. Dhar, H. L. Liu and K. C. Tam, Can. J. Chem. Eng., 2011, 89, 1191-1206.

29 E. Lam, K. B. Male, J. H. Chong, A. C. Leung and J. H. Luong, Trends Biotechnol., 2012, 30, 283-290. 
30 A. Khan, R. A. Khan, S. Salmieri, C. Le Tien, B. Riedl, J. Bouchard, G. Chauve, V. Tan, M. R. Kamal and M. Lacroix, Carbohydr. Polym., 2012, 90, 1601-1608.

31 W. Li, J. Yue and S. Liu, Ultrason. Sonochem., 2012, 19, 479485.
32 Y. Tang, S. Yang, N. Zhang and J. Zhang, Cellulose, 2014, 21, 335-346.

33 I. A. Larmour, S. E. Bell and G. C. Saunders, Angew. Chem., 2007, 119, 1740-1742. 